\title{
Siderophile volatile elements in lunar rocks - constraints on late-stage magmatic degassing
}

\author{
PHILIPP GLEISSNER, JULIE SALME AND HARRY \\ BECKER
}

Freie Universität Berlin

Presenting Author: gleissner@zedat.fu-berlin.de

The volatile element inventory of the Moon and the leading processes of volatile loss and gain are key aspects for models of lunar formation, late accretion and mantle dynamics. Since the era of the Apollo missions, the Moon was thought to be highly depleted in volatile elements relative to Earth and chondrites. This view was recently challenged by studies of pyroclastic glasses and olivine-hosted melt inclusions in such glasses and basalts [1,2], in which elevated concentrations of volatile elements and water were detected. New estimates of the lunar mantle composition suggest only moderate depletion when compared to the bulk silicate Earth [1]. However, in order to balance the proposed model of a less volatile-depleted lunar interior with the strongly volatile-depleted nature of most analyzed lunar samples, strong magmatic degassing is required.

Here we present a different approach to the study of the volatile inventory of magmatic lunar rocks. Mass fractions of siderophile volatile elements (SVE) have been determined by isotope dilution ICP-MS methods from the same aliquot of representative pristine plutonic and volcanic rocks. Our new data for vesicular basalts and non-vesicular basalts and melt inclusion data from the literature [2] display a coherent correlation of $\mathrm{S}$

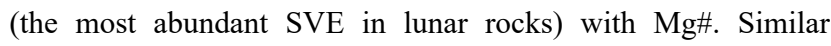
correlations with indicators of fractional crystallization are observed for $\mathrm{Cu}$, the least volatile SVE of this study, and the more volatile elements $\mathrm{Ag}$ and $\mathrm{Cd}$. These observations are entirely consistent with the incompatible behavior of SVE and the notion that lunar mantle-derived magmas did not reach Ssaturation during magmatic differentiation [3]. However, these correlations are in conflict with the proposed strong volatile loss of S and other SVE from such magmas. The results show that the inventory of SVE in most lunar magmatic rocks is controlled by fractional crystallization and much less by magmatic degassing. Furthermore, the extent of volatile loss during magmatic differentiation and formation of mantle source regions is constrained by comparison between samples formed at different depths.

[1] Hauri et al. (2015) EPSL, 409, 252-264.

[2] Day (2018) Am. Min., 103, 1734-1740.

[3] Ni et al. (2019) GCA, 249, 17-41. 